\title{
Iron Efflux from Astrocytes Plays a Role in Remyelination
}

\author{
Katrin Schulz, Antje Kroner, and Samuel David \\ Centre for Research in Neuroscience, The Research Institute of the McGill University Health Center, Montreal, Québec H3G 1A4, Canada
}

How iron is delivered to the CNS for myelination is not well understood. We assessed whether astrocytes can provide iron to cells in the CNS for remyelination. To study this we generated a conditional deletion of the iron efflux transporter ferroportin (Fpn) in astrocytes, and induced focal demyelination in the mouse spinal cord dorsal column by microinjection of lysophosphatidylcholine (LPC). Remyelination assessed by electron microscopy was reduced in astrocyte-specific Fpn knock-out mice compared with wild-type controls, as was proliferation of oligodendrocyte precursor cells (OPCs). Cell culture work showed that lack of iron reduces the ability of microglia to express cytokines (TNF- $\alpha$ and IL-1 $\beta$ ) involved in remyelination. Furthermore, astrocytes in culture express high levels of FGF-2 in response to IL- $1 \beta$, and IGF- 1 in response to TNF- $\alpha$ stimulation. FGF- 2 and IGF- 1 are known to be important for myelination. Reduction in IL-1 $\beta$ and IGF-1 were also seen in astrocyte-specific Fpn knock-out mice after LPC-induced demyelination. These data suggest that iron efflux from astrocytes plays a role in remyelination by either direct effects on OPCs or indirectly by affecting glial activation.

\section{Introduction}

Remyelination in the CNS is primarily performed by oligodendrocyte precursor cells (OPCs; Franklin and ffrench-Constant, 2008). Following demyelination, OPCs proliferate and differentiate into myelinating oligodendrocytes. A number of growth factors have been identified that regulate the proliferation and differentiation phase of OPCs, such as fibroblast growth factor 2 (FGF-2) and insulin-like growth factor 1 (IGF-1; Frost et al., 2003; Hsieh et al., 2004). In addition, cytokines such as tumor necrosis factor- $\alpha$ (TNF- $\alpha$ ) and interleukin- $1 \beta$ (IL-1 $\beta$ ) have also been implicated in remyelination (Arnett et al., 2001; Mason et al., 2001).

Iron is directly involved in the synthesis of myelin as an essential cofactor for enzymes involved in lipid and cholesterol synthesis (Todorich et al., 2009). In addition, iron plays a role in proliferation and differentiation of cells via enzymes involved in energy metabolism and DNA synthesis (Cazzola et al., 1990). Iron deficiency during development leads to hypomyelination (Lozoff and Georgieff, 2006). However, not much is known about the role of iron in remyelination in the adult CNS and how iron is made available to OPCs. Iron is thought to enter the CNS from the circulation via capillary endothelial cells (Moos et al., 2007). As $\sim 95 \%$ of the capillary surface is covered by astrocytic end feet, astrocytes are ideally positioned to take up iron from the circulation and distribute it to other cells in the CNS. Astrocytes

Received 0ct. 21, 2011; revised Jan. 10, 2012; accepted Feb. 11, 2012.

Author contributions: K.S. and S.D. designed research; K.S. and A.K. performed research; K.S. analyzed data; K.S. and S.D. wrote the paper.

This work was supported by a grant from the Canadian Institutes of Health Research (CIHR) to S.D. K.S. has received support from the CIHR Neuroinflammation Training Program and a studentship from the Multiple Sclerosis Society of Canada; A.K. is supported by a CIHR Postdoctoral Fellowship. We thank Dr. Magdalena Götz for providing us with the GLAST::CreERT2 mice and Dr. Nancy Andrews for the Fpn ${ }^{\text {flox/flox }}$ mice. We thank Hiba Kazak for technical assistance and Margaret Attiwell for help with preparing the illustrations.

Correspondence should be addressed to Dr. Samuel David, Centre for Research in Neuroscience, The Research Institute of the McGill University Health Center, Livingston Hall, Room L7-210, 1650 Cedar Avenue, Montreal, Québec H3G 1A4, Canada. E-mail: sam.david@mcgill.ca.

DOI:10.1523/JNEUROSCI.5328-11.2012

Copyright $\odot 2012$ the authors $\quad 0270-6474 / 12 / 324841-07 \$ 15.00 / 0$ possess the iron influx and efflux mechanisms required for cellto-cell transport of iron (Dringen et al., 2007). Iron efflux from astrocytes is mediated by the ubiquitously expressed iron exporter ferroportin (Fpn), which partners with ceruloplasmin (Cp), a ferroxidase that oxidizes ferrous iron $\left(\mathrm{Fe}^{2+}\right)$ transported through Fpn to its ferric form $\left(\mathrm{Fe}^{3+}\right.$; Jeong and David, 2003).

We investigated whether iron released from astrocytes plays a role in remyelination. To study this, we generated mice deficient in astrocytic iron efflux by deleting the iron exporter Fpn specifically in astrocytes and induced demyelination in the spinal cord by injecting lysophosphatidylcholine (LPC) into the dorsal white matter of the spinal cord.

\section{Materials and Methods}

Animals and LPC injections. All procedures were approved by the Animal Care Committee of the Research Institute of the McGill University Health Centre and followed the guidelines of the Canadian Council on Animal Care. Astrocyte-specific conditional deletion of Fpn, the iron efflux transporter, was generated by crossing GLAST::CreERT2 mice (Mori et al., 2006) with Fpn ${ }^{\text {flox/flox }}$ mice (Donovan et al., 2005) to generate GLAST::CreERT2;Fpn ${ }^{\text {flox/flox }}$ mice. Tamoxifen dissolved in corn oil (Sigma)/ethanol (9:1) at $10 \mathrm{mg} / \mathrm{ml}$ and $1 \mathrm{mg}$ was administered intraperitoneally twice a day for $5 \mathrm{~d}$ to $6-8$-week-old female GLAST: CreERT2;Fpn ${ }^{\text {flox/flox }}$ mice. Control female animals of the same genotype were injected with vehicle.

Two weeks after the last tamoxifen injection, GLASTCre: ERT2;Fpn ${ }^{\text {flox/flox }}$ mice or vehicle-injected controls were anesthetized with ketamine/xylazine/acepromazine (100/10/3 mg/kg), a laminectomy performed at the 11th thoracic vertebral level (T11). One microliter of $1 \%$ LPC was injected with a glass micropipette (50 $\mu \mathrm{m}$ tip diameter) into the dorsal column white matter.

PCR genotyping and Western blotting. DNA was extracted from brain, spinal cord, and liver using the DNeasy blood and tissue kit (Qiagen) and PCR was performed using the Advantage 2 PCR Kit (Clontech Laboratories). GLAST::CreERT2;Fpn ${ }^{\text {flox/flox }}$ mice were genotyped with one PCR that had one forward primer ( $5^{\prime}$-CTA CAC GTG CTC TCT TGA GAT- $\left.3^{\prime}\right)$ and two reverse primers (5'-GGT TAA ACT GCT TCA AAG G-3' and 5'-CCT CAT ATG TGA GTC AAA GTA TAG-3'). The Fpn wild-type allele generated a 355 bp band, and the Fpn floxed allele gen- 
A
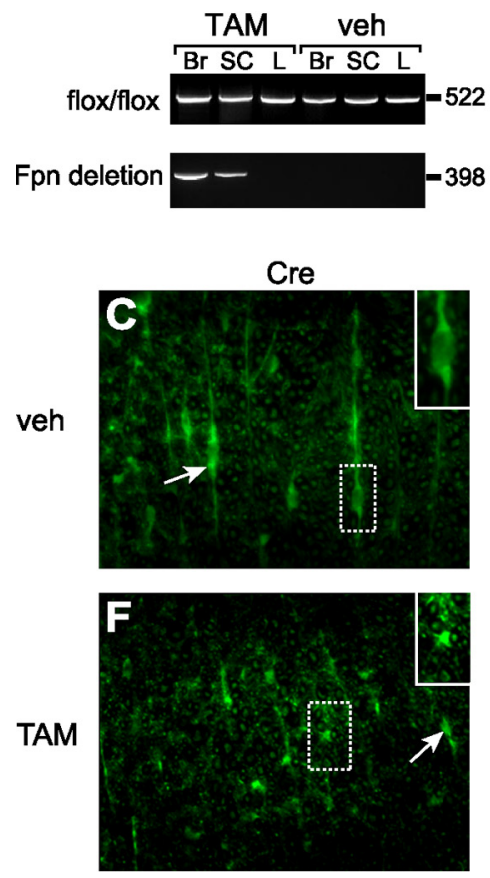

B
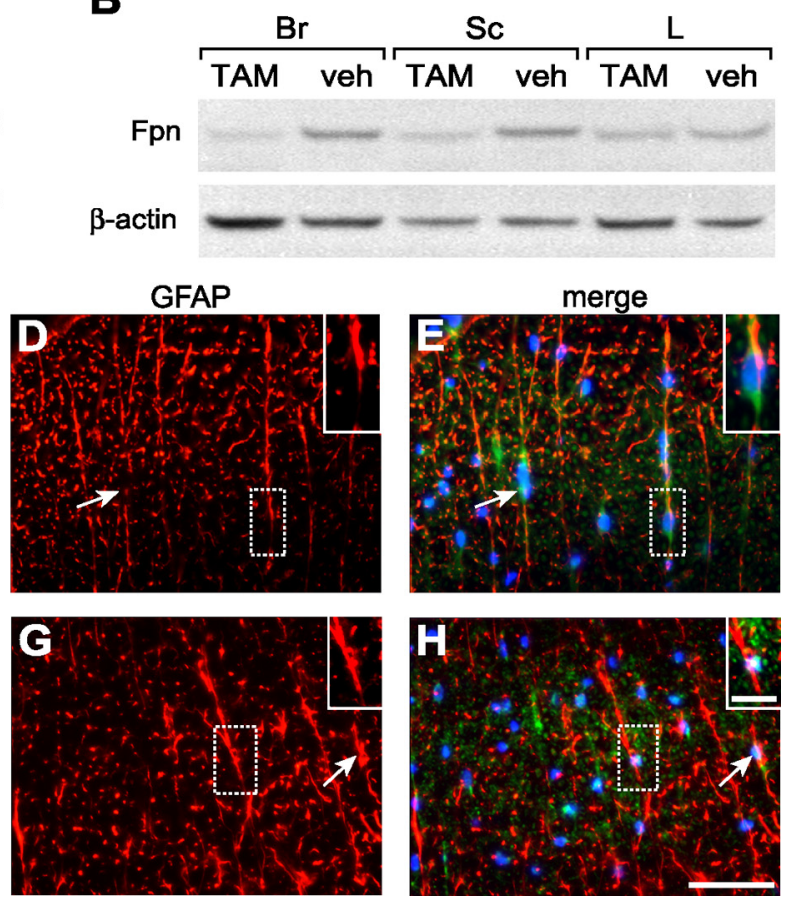

Figure 1. Conditional deletion of Fpn in astrocytes by GLASTCre::ERT2. $\boldsymbol{A}$, The Fpn deletion band (398 bp) is only seen in brain (Br) and spinal cord (SC), but not in the liver (L) of the tamoxifen-treated mouse, nor in the vehicle-treated mouse. The floxed allele ( $522 \mathrm{bp}$ ) is present in all tissues. $\boldsymbol{B}$, Western blot of brain, spinal cord, and liver of GLASTCre:: ERT2; Fpn ${ }^{\text {flox/flox }}$ mice shows reduced Fpn expression in brain and spinal cord but not liver of TAM-treated compared with veh-treated mice. $\boldsymbol{C}-\boldsymbol{H}$, Double immunofluorescence labeling for Cre and GFAP shows the expression of Cre recombinase $(\boldsymbol{C}, \boldsymbol{F})$ in GFAP-positive astrocytes $(\boldsymbol{D}, \boldsymbol{G})$ in TAM- and veh-treated GLASTCre::ERT2;Fpn ${ }^{\text {flox/flox }}$ mice. Note the localization of the Cre recombinase in the cytosol in veh-treated animals $(\boldsymbol{C}-\boldsymbol{E})$, compared with its nuclear localization in TAM-treated animals $(\boldsymbol{F}-\boldsymbol{H})$. Merged images including a DAPI staining to label cell nuclei are shown in $\boldsymbol{E}, \boldsymbol{H}$. Scale bars: (in $\boldsymbol{H}) \boldsymbol{C}-\boldsymbol{H}, 50 \mu \mathrm{m}$; $(\boldsymbol{H}$, inset) $\boldsymbol{C}-\boldsymbol{H}$, insets, $20 \mu \mathrm{m}$.

erated a 522 bp band. The band generated by full deletion of Fpn was 398 bp. Western blotting of protein extracts from spinal cord, brain, and liver tissue using rabbit anti-Fpn IgG (1:1500; Alpha Diagnostics) was performed as described previously (Jeong and David, 2006).

Electron microscopy. Ten days after LPC injections, mice were perfused under deep anesthesia with $0.5 \%$ paraformaldehyde and $2.5 \%$ glutaraldehyde in $0.1 \mathrm{~m}$ phosphate buffer, $\mathrm{pH}$ 7.4. Spinal cord segments containing the lesion site were postfixed overnight in the same fixative and then with $2 \%$ osmium tetroxide for $2 \mathrm{~h}$ and embedded in Epon. Crosssections ( $1 \mu \mathrm{m}$ thick) of the spinal cord were stained with $1 \%$ toluidine blue and examined by light microscopy. Ultrathin sections of the lesion site were stained with lead citrate and viewed with a Philips CM10 electron microscope. The ratio of the axon diameter over the fiber (axon + myelin) diameter (g-ratio) of axons located at the rostrocaudal midpoint of LPC injections was measured from electron micrographs. Briefly, EM images of cross-sections of the lesion site were taken at $1550 \times$ magnification (10-15 images per animal). Because the axons were not always circular, the diameters were calculated from measurements of the axonal circumference and the fiber circumference (i.e., outside the myelin sheath) based on the following formula: $d=p / \pi$ (where $d=$ diameter; $\mathrm{p}=$ perimeter measurement). One hundred axons per animal were analyzed ( $n=3$ animals per group) and statistical significance was determined by two-way repeated-measures (RM) ANOVA.

Immunofluorescence labeling. Animals were perfused with $4 \%$ paraformaldehyde in $0.1 \mathrm{~m}$ phosphate buffer, $\mathrm{pH}$ 7.4. Spinal cord segments containing the lesion site were removed, and processed for cryostat sectioning $(14 \mu \mathrm{m})$ and double immunofluorescence was performed using rat anti-GFAP (1:200; Invitrogen) and rabbit anti-Cre recombinase (1: 400; Covance) or goat anti-olig2 (1:200; R\&D Systems) and rabbit antiKi67 antibody (1:800; Abcam) or rat anti-Mac1 (1:200; Serotec) and goat anti-IL-1 $\beta$ (1:100; R\&D Systems) or rat anti-GFAP (1:200; Invitrogen) and goat anti-IGF-1 (1:100; R\&D Systems) as described previously (Jeong and David, 2006). Images of the IL- $1 \beta / \mathrm{Mac}-1$ and IGF-1/GFAP labeled cells were acquired using a confocal laser scanning microscope
(FluoView FV1000, Olympus) and prepared using FV10-ASW 3.0 software (Olympus). Quantification of the number of IL- $1 \beta$ and IGF-1positive cells in the area of the lesion was estimated from images captured from an Axioskop 2 Plus microscope (Zeiss) using a QImaging Retiga 1300 camera, three sections per animal and three animals $(n=3)$ for each (IL-1 $\beta$ and IGF-1) and extrapolated to square millimeters.

Olig2- and Ki67-double-positive cells or olig2-positive cells were counted at the rostrocaudal midpoint of the lesion and divided by the area of the respective lesion. Counts were obtained from three sections per animal and averaged ( $n=4$ animals per group). Only animals with comparable lesion size were analyzed (mean lesion site CTRL: $0.082 \pm$ $0.001 \mathrm{~mm}^{2}$; KO: $0.085 \pm 0.005 \mathrm{~mm}^{2}$ ).

Cell culture. Microglia cultures were prepared from the cerebral cortex of neonatal mice of either sex as described previously (Saura et al., 2003). Microglial purity examined using Mac-1 immunoreactivity was $\sim 97 \%$. Microglia were incubated with $10 \mathrm{ng} / \mathrm{ml}$ lipopolysaccharide (LPS from Escherichia coli O111:B4) and $100 \mu \mathrm{m}$ of the iron chelator salicylaldehyde isonicotinoyl hydrazone (SIH) overnight. SIH is an analog of pyridoxal isonicotinoyl hydrazone that shows high affinity and selectivity for iron (Richardson and Ponka, 1998). Astrocyte cultures were prepared as described previously (Jeong and David, 2003). To load astrocytes with iron,

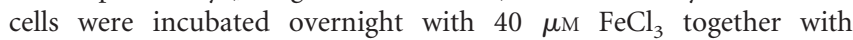
$\mathrm{L}$-ascorbate (molar ratio of $\mathrm{FeCl}_{3}$ to $\mathrm{L}$-ascorbate was 1:44). The next day, astrocytes were either incubated with $10 \mathrm{ng} / \mathrm{ml} \mathrm{TNF}-\alpha$ or $10 \mathrm{ng} / \mathrm{ml} \mathrm{IL}-1 \beta$ overnight.

$R T$-PCR. RNA was isolated from astrocyte and microglia cultures using the RNeasy Minikit (Qiagen) and reverse transcribed to cDNA with the Omniscript Reverse Transcriptase Kit (Qiagen). PCR was performed using the HotStarTaq PCR Kit (Qiagen) with the following primers: TGF- $\beta$ _forward (for): $5^{\prime}$-TGA TAC GCC TGA GTG GCT GTC TTT-3'; TGF- $\beta$ _reverse (rev): $5^{\prime}$-TGT ACT GTG TGT CCA GGC TCC AAA-3'; IGF-1_for: 5' -ACC TCA GAC AGG CAT TGT GGA TGA-3'; IGF-1_rev: 5'-CAG GTT GCT CAA GCA GCA AAG GAT-3'; FGF-2_for: 5'-AAC AGT ATG GCC TTC TGT CCA GGT-3'; FGF-2_rev: 5'-AAG AGC GAC 
A

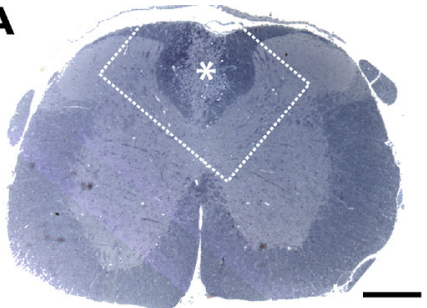

CTRL

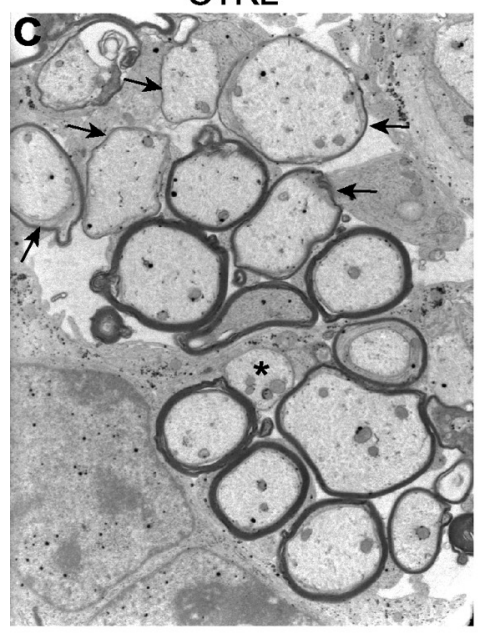

$\mathbf{E}$
B

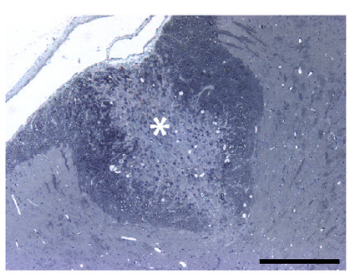

KO

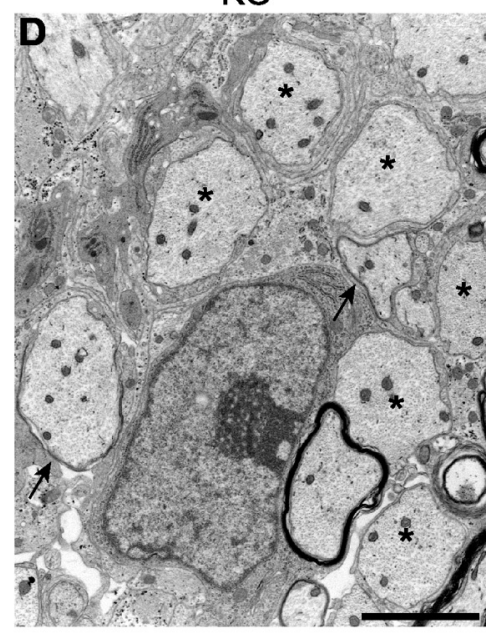

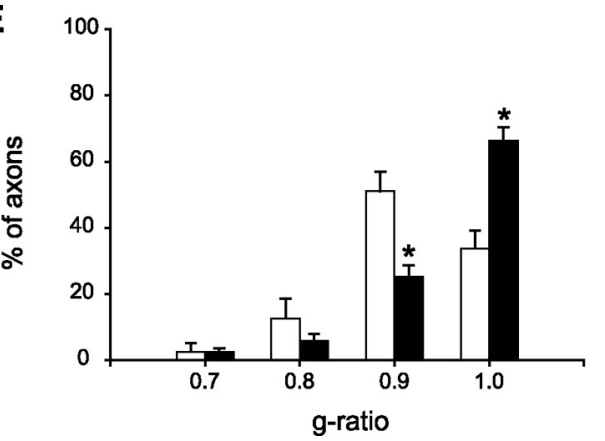

Figure 2. Remyelination after LPC-induced demyelination is impaired in astrocyte-specific Fpn knock-out mice. $\boldsymbol{A}$, Eponembedded cross-section of the spinal cord stained with toluidine blue shows the LPC-induced demyelinating lesion (asterisk) in the dorsal white matter. Scale bar: $300 \mu \mathrm{m}$. B. Higher magnification of the area outlined in the rectangle in $\boldsymbol{A}$. Scale bar: $200 \mu \mathrm{m}$. The demyelinated region is indicated by the asterisk. $C$, Electron micrograph illustrating the extent of remyelination $10 \mathrm{~d}$ after LPCinjection in wild-type mice. $\boldsymbol{D}$, In contrast, remyelination is markedly reduced in astrocyte-specific Fpn KO mice. Scale bar: $3 \mu \mathrm{m}$. $\boldsymbol{E}$, The g-ratio analysis shows a significantly lower percentage of remyelinated axons $(\mathrm{g}$-ratio $=0.9)$ and a significant higher percentage of demyelinated axons ( $\mathrm{g}$-ratio $=1.0$ ) in astrocyte-specific Fpn KO mice compared with wild-type mice $(n=3$, mean \pm SEM; two-way RM ANOVA; $p \leq 0.001)$.

CCA CAC GTC AAA CTA- $3^{\prime} ; I L-1 \beta$ for: $5^{\prime}$-AAG TTT GTC ATG AAT GAT TCC CCT C- $3^{\prime} ; I L-1 \beta \_$rev: $5^{\prime}$-GTC TCA CTA CCT GTG ATG AGT-3'; TNF- $\alpha \_$for: $5^{\prime}$-ATG AGC ACA GAA AGC ATG-3'; TNF- $\alpha \_$rev: $5^{\prime}$-GAA GAC TCC TCC CAG GTA-3'.

\section{Results}

\section{Generation of astrocyte-specific Fpn knock-out mice}

Mice lacking the sole known iron exporter Fpn specifically in astrocytes were generated by using the tamoxifen-inducible CreERT2/loxP system as described in the Materials and Methods section. PCR analysis using primers that selectively recognize the recombined Fpn allele shows Fpn deletion in DNA from brain and spinal cord but not in the liver of tamoxifen-treated animals, nor in vehicle-treated animals (Fig. 1A). The excision of Fpn was further confirmed at the protein level by Western blotting (Fig. $1 B)$. Immunofluorescence staining showed that tamoxifen treat-

ment results in the translocation of Cre recombinase from the cytosol into the nucleus in astrocytes, while it remains localized in the cytosol in vehicle-treated animals (Fig. $1 C-H$ ). Together, these data confirm the excision of Fpn in astrocytes using the tamoxifen-inducible Cre/LoxP system.

\section{Remyelination is impaired in} astrocyte-specific Fpn knock-out mice Injection of LPC into the spinal cord produces focal demyelination within $2-3 \mathrm{~d}$ followed by remyelination starting after 5-7 d (Jeffery and Blakemore, 1995; Ousman and David, 2000). LPC was injected into the dorsal white matter of the spinal cord in astrocyte-specific Fpn KO mice and control mice 2 weeks after the last tamoxifen or vehicle injection. All control animals were GLAST::CreERT2;Fpn $n^{\text {flox/flox }}$ littermates that had been injected intraperitoneally with vehicle instead of tamoxifen. Toluidine blue staining of Epon-embedded spinal cord sections $10 \mathrm{~d}$ after LPC injection shows the lesion site in the dorsal white matter (Fig. 2A,B). Remyelinated axons were identified in electron micrographs by their thin myelin sheath in relation to the axon diameter. Remyelinating axons with thin myelin sheaths were seen in the LPCinjected control animals (Fig. 2C). In contrast, very few remyelinated axons were detected in LPC-injected astrocyte-specific $F p n$ KO mice (Fig. 2D). The percentage of remyelinated axons present in the lesion area was determined by analysis of the $g$-ratio; i.e., the axon diameter divided by the fiber diameter (Foster et al., 1980). A g-ratio of 1 indicates demyelinated or unmyelinated axons, whereas a g-ratio $\geq 0.9$ indicates remyelinated axons. In control animals, $51.03 \pm 6.02 \%$ of the axons within the lesion site show evidence of remyelination and only $33.76 \pm 5.35 \%$ are still demyelinated (Fig. $2 E$ ). In contrast, in the astrocyte-specific Fpn KO mice, only $25.26 \pm 3.53 \%$ of axons are remyelinated and $66.24 \pm 4.22 \%$ are still demyelinated (Fig. $2 E$ ), indicating that remyelination is impaired in the astrocyte-specific Fpn KO mice. These results suggest that iron efflux from astrocytes via Fpn contributes to remyelination.

Proliferation of oligodendrocyte precursor cells is impaired Remyelination involves the proliferation and differentiation of OPCs into myelinating oligodendrocytes. We therefore assessed whether the reduced remyelination seen in astrocyte-specific Fpn $\mathrm{KO}$ mice may be due to a decrease in OPC proliferation. Double immunofluorescence labeling for the proliferation marker Ki67 and the OPC marker olig2 showed a significant reduction in proliferating OPCs in the astrocyte-specific Fpn KO mice compared with control animals (Fig. $3 A-M$ ). The total number of olig2 labeled cells was also significantly reduced (Fig. $3 N$ ). Therefore, 
reduced OPC proliferation may contribute to the impaired remyelination seen in the astrocyte-specific Fpn KO mice. These data also suggest that iron release from astrocytes to OPCs plays a role in proliferation of OPCs after LPC-induced demyelination in vivo.

The expression of TNF- $\alpha$ and IL- $1 \beta$ is reduced in iron-deficient microglia

Macrophages and microglia play an important role in remyelination by clearing myelin debris that is generated during demyelination and by secreting growth factors and cytokines that promote OPC proliferation and differentiation (Kotter et al., 2005; Neumann et al., 2009). The lack of iron efflux from astrocytes in astrocyte-specific Fpn KO mice may deprive not only OPCs but also microglia of iron. We therefore investigated whether iron deficiency alters the ability of microglia to express cytokines. The expression of TNF- $\alpha$ and IL- $1 \beta$, two cytokines implicated in remyelination, was assessed in microglial cultures under iron-deficient conditions. Purified microglia were treated with the iron chelator SIH and stimulated with LPS to induce cytokine expression. LPS-activated microglia show high mRNA expression of $T N F-\alpha$ and $I L-1 \beta$, which are reduced in cells rendered iron-deficient by treating with the iron chelator SIH (Fig. $4 A$ ). These findings indicate that iron is required for the LPS-stimulated expression of TNF- $\alpha$ and IL- $1 \beta$. TNF- $\alpha$ and IL- $1 \beta$ could either directly affect OPC proliferation and differentiation or activate astrocytes to produce growth factors, which could in turn act on remyelinating OPCs. In addition, there is a reduction in IL- $1 \beta$ expression in microglia/ macrophages in the lesion site in astrocytespecific Fpn KO animals $10 \mathrm{~d}$ after LPC injection (Fig. $4 B, D-K$ ).

\section{The expression of growth factors by} astrocytes under high iron conditions We next assessed whether TNF- $\alpha$ and IL- $1 \beta$ can induce astrocytes to express growth factors such as FGF-2 and IGF-1 that promote OPC proliferation and differentiation (Moore et al., 2010). Purified astrocyte cultures were treated with either TNF- $\alpha$ or IL-1 $\beta$ and the mRNA expression of FGF-2 and IGF-1 assessed. The expression of FGF- 2 and $I G F-1$ was significantly upregulated by IL- $1 \beta$ and TNF- $\alpha$, respectively (FGF-2: 3.39-fold $\pm 0.56, p \leq 0.01$; IGF-1: 2.09 -fold $\pm 0.35, p \leq 0.04)$. Therefore, the reduced expression of TNF- $\alpha$ and IL- $1 \beta$ by iron-deprived microglia might result in decreased expression of FGF- 2 and IGF- 1 by astrocytes. TGF- $\beta$ mRNA was also upregulated in astrocyte cultures with IL- $1 \beta$ stimulation (2.96-fold $\pm 0.46, p \leq 0.05$ ) but significantly reduced when astrocytes were iron-loaded $(1.77$-fold $+0.19, p<0.05)$, indicating that iron may be involved in regulating TGF- $\beta$ expression. TGF- $\beta$ is thought to play a role in OPC differentiation
Ki67
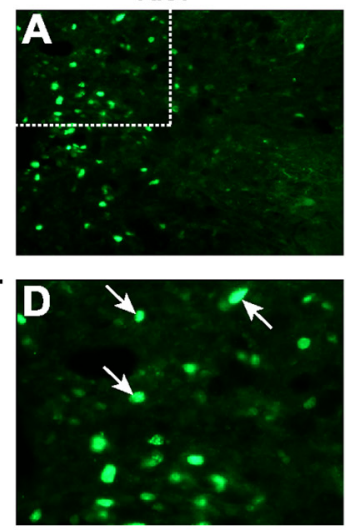

Ki67

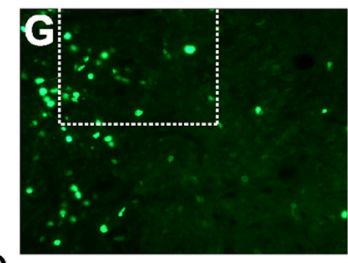

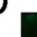

M

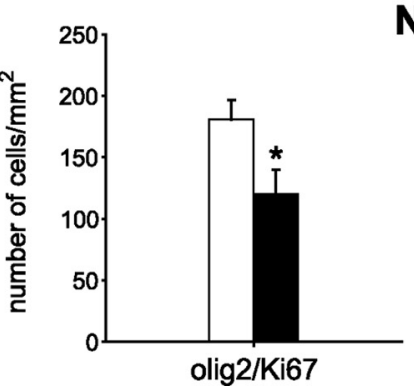

olig2
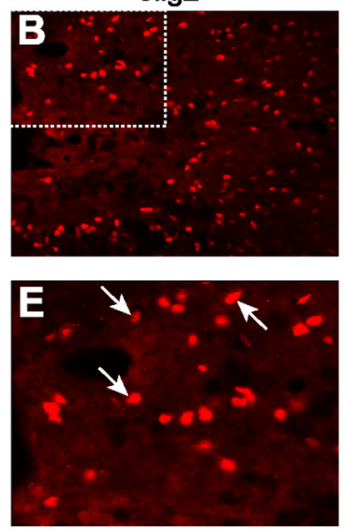

olig2
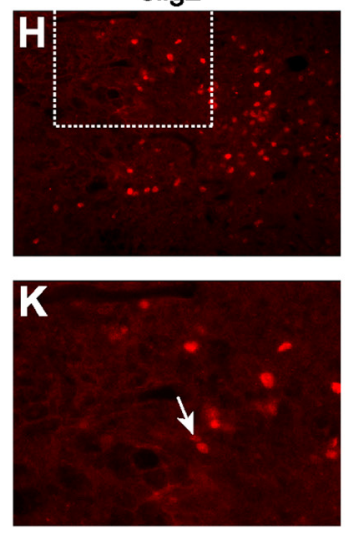

N

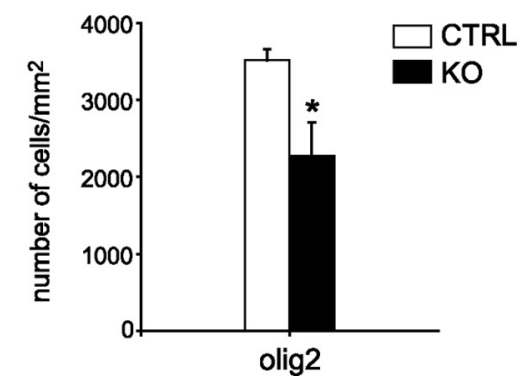

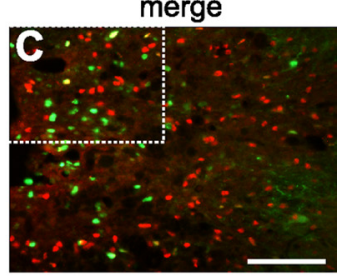
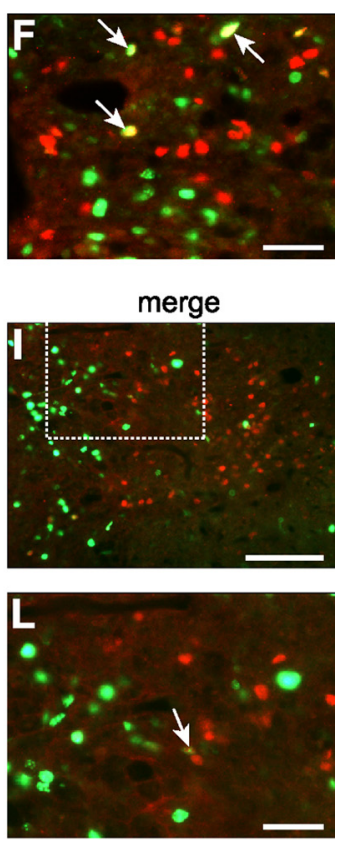

Figure 3. $\mathrm{OPC}$ proliferation after $\mathrm{LPC}$-induced demyelination is reduced in astrocyte-specific Fpn knock-out mice. Double immunofluorescence labeling for the proliferation marker Ki67 $(\boldsymbol{A}, \boldsymbol{D}, \boldsymbol{G}, \boldsymbol{J})$ and the $\mathrm{OPC}$ marker olig2 $(\boldsymbol{B}, \boldsymbol{E}, \boldsymbol{H}, \boldsymbol{K})$ shows a reduction in proliferating $\mathrm{OPC}$ in astrocyte-specific $F p n \mathrm{KO}$ mice $(\boldsymbol{G}-\boldsymbol{L})$ compared with control mice $(\boldsymbol{A}-\boldsymbol{F})$; arrows indicate double-labeled cells. Merged images are shown in $\mathbf{C}, \boldsymbol{F}, \boldsymbol{I}, \boldsymbol{L}$. Scale bars: (in $\boldsymbol{C}, \boldsymbol{I}) \boldsymbol{A}-\boldsymbol{C}, \boldsymbol{G}-\boldsymbol{I}, 100 \mu \mathrm{m}$; (in $\boldsymbol{F}, \boldsymbol{L}) \boldsymbol{D}-\boldsymbol{F}, \boldsymbol{J}-\boldsymbol{L}, 50 \mu \mathrm{m} . \boldsymbol{M}, \boldsymbol{N}$, Quantification OPCS $(\boldsymbol{M})$ and in total numbers of OPCS $(\boldsymbol{N})$ in the area of LPC-induced demyelination in astrocyte-specific Fpn KO mice compared with wild-type mice $(n=4$; mean \pm SEM; Student's $t$ test; $p \leq 0.05)$.

(McKinnon et al., 1993). Furthermore, there is a reduction in IGF-1 expression in astrocytes in the lesion site in astrocytespecific Fpn $\mathrm{KO}$ animals $10 \mathrm{~d}$ after LPC injection (Fig. 4C,L-S).

The reduced remyelination in astrocyte-specific Fpn KO mice might therefore be due to direct effects on OPCs through limited iron supply or to indirect effects via iron-deficient microglia, which could in turn affect astrocyte expression of growth factors involved in OPC proliferation and differentiation as summarized in Figure $4 T$.

\section{Discussion}

Our results revealed impaired remyelination in astrocyte-specific $\mathrm{Fpn} \mathrm{KO}$ mice, suggesting that iron efflux from astrocytes plays a role in CNS remyelination. The defects in remyelination could be 
A

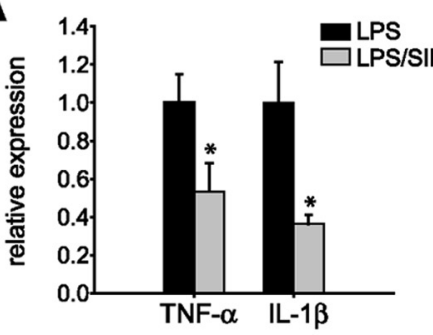

B
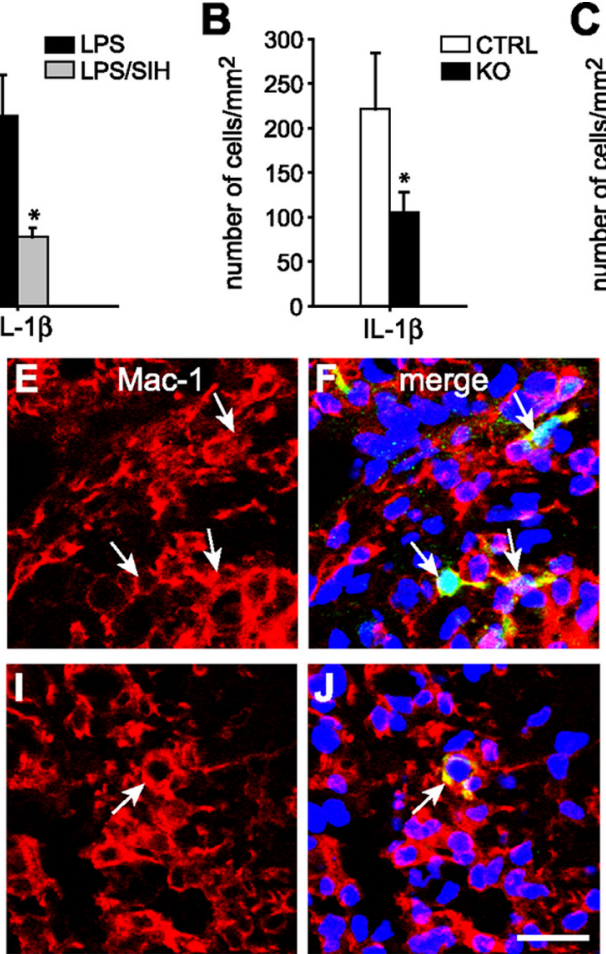
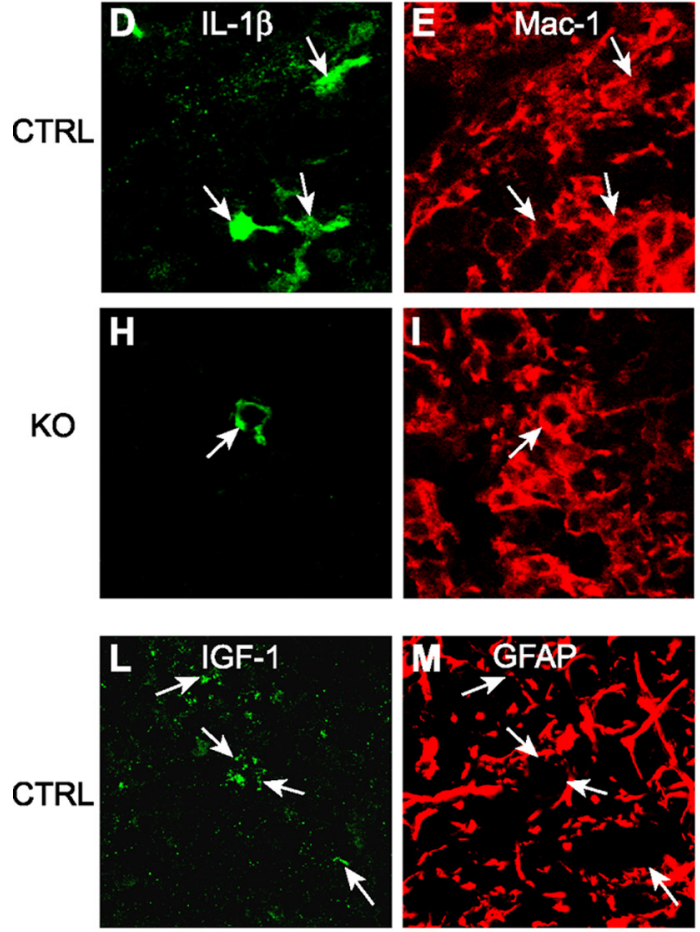

C
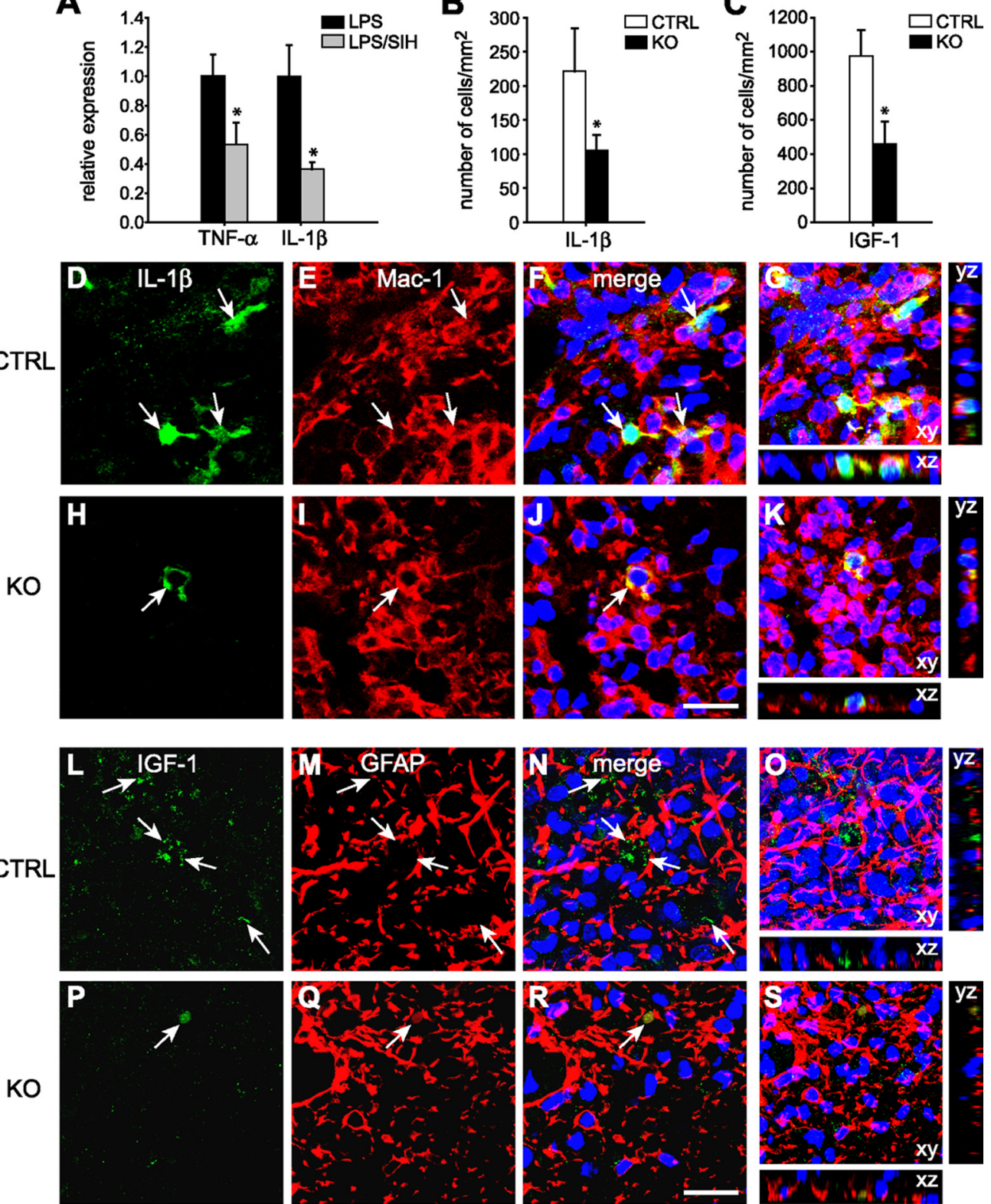

T Fpn KO astrocyte

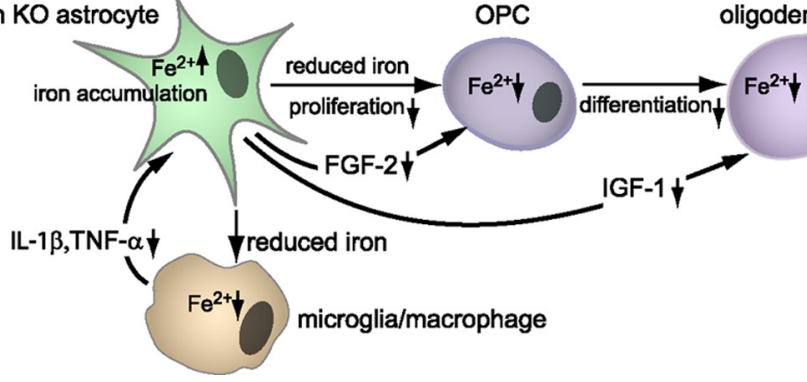

Figure 4. Effects of blocking iron efflux from astrocytes on growth factor expression during remyelination. $A, R T-P C R$ analysis demonstrates a significant reduction in TNF- $\alpha$ and IL- $1 \beta$ mRNA expression in LPS-stimulated microglia in vitro treated with the iron chelator SIH compared with vehicle (mean \pm SEM; Student's $t$ test; $p \leq 0.05 ; n=3$ ). $B, C$, Quantification shows a significant reduction in the numbers of IL-1 $\beta+$ cells $(\boldsymbol{B})$ and IGF-1+ cells $(\boldsymbol{C})$ in the LPC lesion site in astrocyte-specific Fpn K0 mice compared with wild-type control (CTRL) mice (three sections per animal; $n=3$ mice per group; mean \pm SEM; Student's $t$ test; $p \leq 0.04)$. $\boldsymbol{D}-\boldsymbol{F}$, Single-plane confocal images ( $0.6 \mu \mathrm{m}$ thickness) of double immunofluorescence labeling of control mice showing IL-1 $\beta$ labeling ( $\boldsymbol{D}, \boldsymbol{F}$, arrows) and Mac-1 labeling for macrophage/microglia $(\boldsymbol{E}, \boldsymbol{F}$, arrow). $\boldsymbol{G}, \mathrm{A}$ full $z$-stack image (14 $\mu \mathrm{m})$ showing double labeling for IL-1 $\beta$ (green) and Mac- 1 (red) in the $x-y, x-Z$, and $y$-z planes. $\boldsymbol{H}-\boldsymbol{J}$, Single-plane confocal images of astrocyte-specific Fpn KO mice showing reduced labeling for IL-1 $\beta$ ( $\boldsymbol{H}, \boldsymbol{J}$, arrows) in Mac-1 labeled cells $(\boldsymbol{I}, \boldsymbol{J}$, arrows) compared with the control mice shown in $\mathbf{D}-\boldsymbol{G}$. $\boldsymbol{K}$, A full $z$-stack image showing double labeling for IL-1 $\beta$ (green) and Mac- 1 (red) in the $\mathbf{x}-\mathbf{y}, \mathbf{x}-\mathbf{z}$, and $y-z$ planes. $\boldsymbol{F}, \mathbf{G}, \mathbf{J}, \boldsymbol{K}$ show merged images including DAPI labeling of the nuclei. $\boldsymbol{L}-\boldsymbol{N}$, Single plane confocal images of control mice showing IGF-1 $\left(\boldsymbol{L}, \boldsymbol{N}\right.$, arrows) expression in GFAP ${ }^{+}$astrocytes $(\boldsymbol{M}, \boldsymbol{N}), \mathbf{0}, \mathrm{A}$ full $\mathbf{z}$-stack image showing double labeling for IGF- 1 (green) and GFAP (red) in the $x-y, x-z$, and $y-z$ planes. As is to be expected, the GFAP labeling of the intermediate filaments surrounds or lies adjacent to the IGF- 1 labeling. $\boldsymbol{P}-\boldsymbol{R}$, Single-plane confocal images of astrocyte-specific Fpn KO mice showing reduced labeling for IGF- $1\left(\boldsymbol{P}, \boldsymbol{R}\right.$, arrows) in GFAP ${ }^{+}$astrocytes $(\boldsymbol{Q}, \boldsymbol{R}$, arrows) compared with the control mice shown in $\boldsymbol{L}-\mathbf{0}$. S, A full $\boldsymbol{Z}$-stack image showing double labeling for IGF-1 and GFAP in the $x-y, x-z$, and $y-z$ planes. $\boldsymbol{N}, \mathbf{0}, \boldsymbol{R}$, S show merged images including DAPI labeling of the nuclei. Scale bars: $20 \mu \mathrm{m}$. $\boldsymbol{T}$, Schematic (Figure legend continues.) 
due to decreased proliferation and/or differentiation of OPCs. Iron is known to be required for proliferation and differentiation of cells, because it plays an important role in DNA synthesis as well as in oxidative metabolism (Cazzola et al., 1990). Iron has also been shown to be involved in proliferation of OPCs in vitro (Morath and Mayer-Pröschel, 2001) and in oligodendrogenesis after intraspinal LPS injections (Schonberg and McTigue, 2009). Since iron would be retained within astrocytes in the astrocytespecific Fpn KO animals, OPCs might be iron-deficient, which may account for the reduced proliferation of OPCs after LPCinduced demyelination in these mice. Our in vivo results suggest that iron efflux from astrocytes contributes to OPC proliferation. Microglia/macrophages have also been suggested to deliver iron to OPCs for oligodendrocyte genesis after intraspinal LPS injection (Schonberg and McTigue, 2009). However, in the LPS model, increased iron accumulation was detected in macrophages at the lesion, whereas no iron accumulation was observed in the LPC-induced demyelination model used in the current study, suggesting that astrocytes rather than microglia might be a major source of iron in the LPC model. The LPS-induced iron accumulation in microglia/macrophages may be a reflection of the response of macrophages to sequester iron in response to bacterial infection (Nairz et al., 2011).

In addition to the direct effects of iron deprivation on OPCs, iron may also have indirect effects on remyelination, such as altering the levels of cytokines and growth factors that can influence OPC proliferation and differentiation. Iron retention in astrocytes in the astrocyte-specific Fpn KO mice could also render microglia iron-deficient, which might affect their activation state and cytokine expression. Cytokines such as TNF- $\alpha$ and IL-1 $\beta$ have been shown to play a role in remyelination (Arnett et al., 2001; Mason et al., 2001). We found here that LPS-stimulated microglia in cell culture show decreased expression of TNF- $\alpha$ and IL- $1 \beta$ when treated with an iron chelator to render them irondeficient. These data suggest a role of iron in the expression of TNF- $\alpha$ and IL- $1 \beta$ by microglia, which is consistent with a previous report showing decreased expression of these cytokines by microglia treated with the iron chelator deferoxamine (Zhang et al., 2006). TNF- $\alpha$ has been shown to directly promote OPC proliferation and remyelination through its receptor TNFR2 (Arnett et al., 2001). In addition, TNF- $\alpha$ and IL- $1 \beta$ may also have indirect effects by activating other cells within the CNS to produce growth factors involved in OPC proliferation and differentiation (Mason et al., 2001). One of the factors known to induce OPC proliferation is FGF-2 (Wolswijk and Noble, 1992). FGF-2 is predominantly expressed by astrocytes in response to myelin damage (Messersmith et al., 2000). Furthermore, it is also upregulated in reactive astrocytes after LPC-induced demyelination (Hinks and Franklin, 1999). We found that stimulation of astrocytes with IL- $1 \beta$ induced increased expression of FGF-2. TNF- $\alpha$ has a similar effect on IGF-1 expression. IGF-1 not only promotes OPC differentiation and survival but also affects OPC proliferation

\section{$\leftarrow$}

(Figure legend continued.) summarizing the direct and indirect effects of blocking iron efflux from astrocytes on remyelination. Astrocytes in the Fpn-conditional null mice retain iron due to deletion of the iron exporter $F p n$. Iron from astrocytes thus cannot be made available to other cells in the CNS. This reduced iron availability can directly affect the proliferation and differentiation of OPCs. In addition, microglia/macrophages might also be iron-deficient in the astrocyte-specific Fpn-null mice, which can impair their ability to express cytokines such as IL-1 $\beta$ and TNF- $\alpha$. These cytokines are indirectly implicated in remyelination by stimulating astrocytes to produce growth factors involved in OPC proliferation (FGF-2) and differentiation (IGF-1).
(Mozell and McMorris, 1991). IGF-1 is also expressed by astrocytes (Ballotti et al., 1987) and plays a protective and regenerative role after experimentally induced demyelination (Mason et al., 2000). These results suggest that FGF-2 and IGF-1 can be upregulated by IL- $1 \beta$ and TNF- $\alpha$, respectively. We further showed that IL- $1 \beta$ expression by microglia/macrophages, as well as IGF- 1 expression by astrocytes, was also reduced in the LPC lesion site of astrocyte-specific Fpn KO mice, which may contribute to impaired OPC proliferation and differentiation. TGF- $\beta$ has been suggested to play a role in differentiation of OPCs and is also upregulated after LPC-induced demyelination (Hinks and Franklin, 1999). We show here that IL- $1 \beta$-stimulated astrocytes upregulate TGF- $\beta$ expression, which is markedly reduced when astrocytes are iron-loaded.

In summary, our findings suggest that iron efflux from astrocytes through the iron exporter Fpn plays a role in providing iron for remyelination. Since iron may also be available from other sources, the deletion of astrocyte Fpn may delay remyelination rather than block it. Additional work will be needed to clarify this. As illustrated in Figure $4 N$, astrocytes may acquire iron from brain capillary endothelial cells via their astrocytic end feet. Release of iron from astrocytes would make it available to OPCs, which can then use the iron for enzymatic processes involved in their proliferation and differentiation into myelin-forming cells. In addition, astrocytes might also provide iron to microglia, which require iron for the expression of IL- $1 \beta$ and TNF- $\alpha$. These two cytokines could then act either directly on OPCs or stimulate astrocytes to produce growth factors involved in OPC proliferation and differentiation such as FGF- 2 and IGF-1. IL-1 $\beta$ can also induce TGF- $\beta$ expression by astrocytes, which could contribute directly to OPC differentiation. These results suggest an important role for astrocytes in taking up iron from endothelial cells at the blood-brain barrier and distributing it to other cell types in the CNS.

\section{References}

Arnett HA, Mason J, Marino M, Suzuki K, Matsushima GK, Ting JP (2001) TNF alpha promotes proliferation of oligodendrocyte progenitors and remyelination. Nat Neurosci 4:1116-1122.

Ballotti R, Nielsen FC, Pringle N, Kowalski A, Richardson WD, Van Obberghen E, Gammeltoft S (1987) Insulin-like growth factor I in cultured rat astrocytes: expression of the gene, and receptor tyrosine kinase. EMBO J 6:3633-3639.

Cazzola M, Bergamaschi G, Dezza L, Arosio P (1990) Manipulations of cellular iron metabolism for modulating normal and malignant cell proliferation: achievements and prospects. Blood 75:1903-1919.

Donovan A, Lima CA, Pinkus JL, Pinkus GS, Zon LI, Robine S, Andrews NC (2005) The iron exporter ferroportin/Slc40al is essential for iron homeostasis. Cell Metab 1:191-200.

Dringen R, Bishop GM, Koeppe M, Dang TN, Robinson SR (2007) The pivotal role of astrocytes in the metabolism of iron in the brain. Neurochem Res 32:1884-1890.

Foster RE, Kocsis JD, Malenka RC, Waxman SG (1980) Lysophosphatidyl choline-induced focal demyelination in the rabbit corpus callosum. Electron-microscopic observations. J Neurol Sci 48:221-231.

Franklin RJ, ffrench-Constant C (2008) Remyelination in the CNS: from biology to therapy. Nat Rev Neurosci 9:839-855.

Frost EE, Nielsen JA, Le TQ, Armstrong RC (2003) PDGF and FGF2 regulate oligodendrocyte progenitor responses to demyelination. J Neurobiol 54:457-472.

Hinks GL, Franklin RJ (1999) Distinctive patterns of PDGF-A, FGF-2, IGF-I, and TGF-betal gene expression during remyelination of experimentally-induced spinal cord demyelination. Mol Cell Neurosci 14:153-168.

Hsieh J, Aimone JB, Kaspar BK, Kuwabara T, Nakashima K, Gage FH (2004) IGF-I instructs multipotent adult neural progenitor cells to become oligodendrocytes. J Cell Biol 164:111-122. 
Jeffery ND, Blakemore WF (1995) Remyelination of mouse spinal cord axons demyelinated by local injection of lysolecithin. J Neurocytol 24:775-781.

Jeong SY, David S (2003) Glycosylphosphatidylinositol-anchored ceruloplasmin is required for iron efflux from cells in the central nervous system. J Biol Chem 278:27144-27148.

Jeong SY, David S (2006) Age-related changes in iron homeostasis and cell death in the cerebellum of ceruloplasmin-deficient mice. J Neurosci 26:9810-9819.

Kotter MR, Zhao C, van Rooijen N, Franklin RJ (2005) Macrophagedepletion induced impairment of experimental CNS remyelination is associated with a reduced oligodendrocyte progenitor cell response and altered growth factor expression. Neurobiol Dis 18:166-175.

Lozoff B, Georgieff MK (2006) Iron deficiency and brain development. Semin Pediatr Neurol 13:158-165.

Mason JL, Jones JJ, Taniike M, Morell P, Suzuki K, Matsushima GK (2000) Mature oligodendrocyte apoptosis precedes IGF-1 production and oligodendrocyte progenitor accumulation and differentiation during demyelination/remyelination. J Neurosci Res 61:251-262.

Mason JL, Suzuki K, Chaplin DD, Matsushima GK (2001) Interleukin1beta promotes repair of the CNS. J Neurosci 21:7046-7052.

McKinnon RD, Piras G, Ida JA Jr, Dubois-Dalcq M (1993) A role for TGFbeta in oligodendrocyte differentiation. J Cell Biol 121:1397-1407.

Messersmith DJ, Murtie JC, Le TQ, Frost EE, Armstrong RC (2000) Fibroblast growth factor 2 (FGF2) and FGF receptor expression in an experimental demyelinating disease with extensive remyelination. J Neurosci Res 62:241-256.

Moore CS, Abdullah SL, Brown A, Arulpragasam A, Crocker SJ (2011) How factors secreted from astrocytes impact myelin repair. J Neurosci Res 89:13-21.

Moos T, Rosengren Nielsen T, Skjørringe T, Morgan EH (2007) Iron trafficking inside the brain. J Neurochem 103:1730-1740.

Morath DJ, Mayer-Pröschel M (2001) Iron modulates the differentiation of a distinct population of glial precursor cells into oligodendrocytes. Dev Biol 237:232-243

Mori T, Tanaka K, Buffo A, Wurst W, Kühn R, Götz M (2006) Inducible gene deletion in astroglia and radial glia-a valuable tool for functional and lineage analysis. Glia 54:21-34.

Mozell RL, McMorris FA (1991) Insulin-like growth factor I stimulates oligodendrocyte development and myelination in rat brain aggregate cultures. J Neurosci Res 30:382-390.

Nairz M, Schroll A, Sonnweber T, Weiss G (2010) The struggle for iron-a metal at the host-pathogen interface. Cell Microbiol 12:1691-1702.

Neumann H, Kotter MR, Franklin RJ (2009) Debris clearance by microglia: an essential link between degeneration and regeneration. Brain 132:288-295.

Ousman SS, David S (2000) Lysophosphatidylcholine induces rapid recruitment and activation of macrophages in the adult mouse spinal cord. Glia 30:92-104.

Richardson DR, Ponka P (1998) Pyridoxal isonicotinoyl hydrazone and its analogs: potential orally effective iron-chelating agents for the treatment of iron overload disease. J Lab Clin Med 131:306-315.

Saura J, Tusell JM, Serratosa J (2003) High-yield isolation of murine microglia by mild trypsinization. Glia 44:183-189.

Schonberg DL, McTigue DM (2009) Iron is essential for oligodendrocyte genesis following intraspinal macrophage activation. Exp Neurol 218:64-74

Todorich B, Pasquini JM, Garcia CI, Paez PM, Connor JR (2009) Oligodendrocytes and myelination: the role of iron. Glia 57:467-478.

Wolswijk G, Noble M (1992) Cooperation between PDGF and FGF converts slowly dividing $\mathrm{O}-2 \mathrm{~A}^{\text {adult }}$ progenitor cells to rapidly dividing cells with characteristics of $\mathrm{O}-2 \mathrm{~A}^{\text {perinatal }}$ progenitor cells. J Cell Biol 118:889-900.

Zhang X, Surguladze N, Slagle-Webb B, Cozzi A, Connor JR (2006) Cellular iron status influences the functional relationship between microglia and oligodendrocytes. Glia 54:795-804. 